\title{
Models in Search of Targets: Exploratory Modelling and the Case of Turing Patterns
}

\author{
Axel Gelfert \\ Department of Philosophy, Literature, History of Science \& Technology \\ Technical University of Berlin \\ Straße des 17. Juni 135, 10623 Berlin \\ email: axel@gelfert.net
}

\begin{abstract}
Traditional frameworks for evaluating scientific models have tended to downplay their exploratory function; instead they emphasize how models are inherently intended for specific phenomena and are to be judged by their ability to predict, reproduce, or explain empirical observations. By contrast, this paper argues that exploration should stand alongside explanation, prediction, and representation as a core function of scientific models. Thus, models often serve as starting points for future inquiry, as proofs of principle, as sources of potential explanations, and as a tool for reassessing the suitability of the target system (and sometimes of whole research agendas). This is illustrated by a case study of the varied career of reactiondiffusion models in the study of biological pattern formation, which was initiated by Alan Turing in a classic 1952 paper. Initially regarded as mathematically elegant, but biologically irrelevant, demonstrations of how, in principle, spontaneous pattern formation could occur in an organism, such Turing models have only recently rebounded, thanks to advances in experimental techniques and computational methods. The long-delayed vindication of Turing's initial model, it is argued, is best explained by recognizing it as an exploratory tool (rather than as a purported representation of an actual target system).
\end{abstract}

\section{Introduction}

It is a recurring feature of contemporary philosophical writing on scientific models that it begins by recognizing their heterogeneous and diverse nature. "Many different things", we are told, "can serve as models including physically constructed scale models, model organisms, and mathematical objects such as sets of trajectories through a state-space" (Weisberg, 2007, pp. 216-217). It has been argued persuasively that, in spite of such diversity, models are instruments of inquiry in their own right, enjoying partial autonomy from both theory and data. Thus understood, models are neither mere approximations to an 'underlying' fundamental theory, nor are they simply a convenient 'shorthand' for aggregating observations. As Margaret Morrison and Mary Morgan put it, models, in a 
manner of speaking, are located "outside the theory-world axis" (Morrison \& Morgan, 1999, p. 17); their construction "involves a complex activity of integration" (Morrison, 1999, p. 44). While attention to the specifics of case studies has vastly improved our understanding of what models are, and how they are being constructed, when it comes to their evaluation and testing, more traditional criteria are typically being invoked. Thus, models are thought to be "inherently intended for specific phenomena" (Suárez, 1999, p. 75) and, importantly, are to be judged by their ability to predict, reproduce, or explain observational data. Indeed, in contexts that require selecting one of a set of candidate models, classical hypothesis-testing remains an important 'intuition pump' for how we ought to assess choices in modelling (e.g. (Steele \& Werndl, forthcoming)), thereby subsuming the assessment of models under wellunderstood - but ultimately limiting - frameworks for the evaluation of theoretical hypotheses.

In this chapter, I shall argue that traditional frameworks for evaluating models have tended to downplay their exploratory function; or, to give my thesis a more positive spin, I shall make the case that exploration should stand alongside explanation, prediction, and representation as a core function of scientific models. This is not to deny that, often, scientific models are intended for specific observed phenomena, nor is it to claim that all modelling must be inherently exploratory; rather, it is intended to draw attention to the - largely overlooked - role of models and modelling in the context of exploratory science. Given the complementary nature of the goal of establishing exploration alongside (rather than in place of) the more traditional functions of scientific models, the main focus of this chapter is to bring out with as much as clarity as possible what we stand to gain from admitting exploration as a criterion for the evaluation of models. The overall thrust of the present paper, thus, is a constructive one, and little space will be devoted to the critique of more traditional criteria of assessment. (A comprehensive survey and critique of traditional accounts of modelling, along with a proposal of a more practice-oriented functional approach, is given in (Gelfert, 2016).) When formulated at this level of generality, the goal of establishing models as - at least in part - exploratory tools in science might seem vague and unspecific. At the very least, it calls for significant clarification, which is what the next two sections will attempt. Section 2 ('Exploration, heuristics, and the (im)possibility of "theory-free" science') positions the present paper in relation to the - by now, well-established - philosophical debate about exploratory experimentation, and tries to demarcate the notion of 'exploration' from the concept of 'heuristics', which has been the topic of a long-standing debate about the place of heuristic reasoning in science. In particular, it is argued that exploratory inquiry exhibits structure and is characterized by recurring strategies, both of which lend themselves to analysis and assessment in terms of their adequacy and fruitfulness. Section 3 ('Functions and uses of exploratory models') follows recent analyses by distinguishing between four main exploratory uses of models: as starting points for future inquiry, as proofs of principle, as sources of potential explanations, and as a tool for reassessing the suitability of the target system. ${ }^{1}$ These four aspects of exploratory modelling are neither intended to be exhaustive, nor are they mutually exclusive. Indeed, as I shall argue, the role of exploratory models as proofs of principle can be understood as addressing both methodological issues and

\footnotetext{
${ }^{1}$ Section 3 follows my discussion in (Gelfert, 2016, pp. 71-99).
} 
explanatory desiderata. Sometimes what needs to be demonstrated is the feasability of a particular (e.g., mathematical) approach, whereas on other occasions the proof of principle consists in specifying, by way of example, a 'how-possibly' explanation.

Section 4 ('The case of reaction-diffusion models for biological pattern formation') undertakes a detailed case study of how the various functions of exploratory modelling interact, and work out, in practice. This is illustrated by the varied career of reaction-diffusion models in the study of biological pattern formation. First proposed by Alan Turing in 1952, the basic idea is that pattern formation in biological systems may arise from the interplay of two chemical substances ('morphogens'), one of which is locally activated, while the other gives rise to long-range inhibition, with both differing in their diffusion rates. As a result, such Turing models predict distinct spatial patterns of different 'chemical wavelengths', corresponding to the concentration of the two morphogens, which in turn may trigger the expression of different phenotypes. Initially regarded as a mathematically elegant, but biologically irrelevant, proof of how, in principle, spontaneous pattern formation could occur, the model was subsequently obscured by other, more intuitively representational and empirically well-supported models. Only recently, due in part to the development of better computer simulation techniques, has the Turing model rebounded and has come to be recognized as contributing to our understanding of real-world cases of biological pattern formation. Exploration, then, holds out the promise of, but neither requires nor guarantees, long-term applicability of a model to specific real-world target systems.

\section{Exploration, heuristics, and the (im)possibility of 'theory-free' science}

On what is little more than a caricature view of the scientific method, science progresses through a series of bold hypotheses, from which predictions are deduced which are subsequently put to the test, leading us to give up our hypotheses when the predictions are found to be flawed, or to hold on to them - albeit only tentatively - for as long as our hypotheses pass whatever empirical tests we subject them to. The limitations, both descriptive and normative, of such extreme hypothetico-deductivism have, of course, long been a topic of philosophical discussion, and one would be hard-pressed, in this day and age, to find bona fide hypothetico-deductivists in large numbers. Yet, elements of this view have proved remarkably stubborn, not least the idea that the only way to assess science is by looking at its past track record of predictions and explanations (rather than, say, by developing a measure of what, following Thomas Nickles, one might call its "generative potential" (Nickles, 1985, p. 184)). Furthermore, testing - that is, the practice of bringing about circumstances that allow researchers to compare reality against previously derived, empirically observable consequences of a theory - becomes the gold standard for evaluating hypotheses, thereby neglecting the fact that "scientists sometimes reason their way towards hypotheses" and not "from hypotheses" towards testable conclusions (Hanson, 1960, p. 105).

The issue has traditionally - sometimes misleadingly - been characterized in terms of Hans Reichenbach's distinction between the "context of discovery" and the "context of 
justification", with only the latter being deemed capable of being rationally assessed. Research activities that precede the explicit formulation of hypotheses and theories, on this caricature view, would be considered to be of merely auxiliary value, subject to psychological, social, and other circumstantial factors which might perchance lead a scientist to stumble upon an improbable, yet ultimately fruitful, idea, but which could not easily be generalized to result in methodological guidelines for what constitutes good science. It goes (almost) without saying that this stark opposition between a (rationally assessable) "context of justification" and an (unruly and arbitrary) "context of discovery" is philosophically indefensible. As numerous authors have argued, it is based on conflating various aspects of scientific practice and its philosophical analysis. For one, it equates 'discovery' with the generation of novel ideas, as in the much-cited example of Friedrich August Kekulé's (possibly apocryphal) daydream of the snake eating its own tail, which led him to hypothesize the novel chemical structure of the benzene ring. Yet, as Theodore Arabatzis has forcefully argued, a mere novel hypothesis - even if it later turns out to be true - does not yet qualify as a discovery, since 'discovery' is a success term and, as such, needs to be grounded in justificatory activity: 'The context of discovery is 'laden' with the context of justification because 'discovery' is a term which refers to an epistemic achievement: if one succeeds in discovering something then, no doubt, this something exists." (Arabatzis, 2006, p. 217) Instead of a binary distinction between two contexts, what one finds, then, is a spectrum of perspectives, each highlighting different (normative and descriptive) aspects and desiderata; any attempt to tease these apart will lead not to a single, but to a multiplicity of distinctions. ${ }^{2}$

An overly narrow view of the relation between discovery and justification would be especially restrictive when turning one's attention to the early stages of scientific inquiry, more specifically: to exploratory modes of inquiry. Labelling an episode of scientific research 'exploratory' is intended to convey more than just a sense of its priority in the chronological order of events. Rather, the label 'exploratory' pertains to a particular mode of doing science: one that aims at getting a grasp of a phenomenon or scientific problem in the absence of a well-understood and workable theory of the domain in question. For a theory to be well-understood and workable, it is not, of course, required that it be true, nor that it must aim for a reductionist account of whatever phenomenon is under investigation. Rather, the idea is that, in those cases that have traditionally received the most attention in philosophy of science, a significant prior body of theoretical knowledge can be assumed to be available, which in turn suggests - not by itself, of course, but in the hands of able scientists - a way of rendering the phenomenon theoretically tractable, at least in principle. By contrast, in exploratory research the existence of such a body of theoretical knowledge cannot be assumed, or is itself at issue. This means that, as analysts of the scientific process, we cannot readily turn to familiar patterns of making sense of what is going on in terms of applying theoretical knowledge to a particular case (or a particular class of cases).

Nowhere is this more obvious than in cases of exploratory experimentation, which have garnered considerable interest from historians and philosophers of science over the past twenty years or so. In 1997, Friedrich Steinle and Richard Burian, independently of one

\footnotetext{
${ }^{2}$ Paul Hoyningen-Huene, in a much-cited paper, distinguishes between five different versions of how one might contrast discovery and justification in scientific practice. See (Hoyningen-Huene, 2006).
} 
another, published papers arguing for 'exploratory experimentation' as a distinctive mode of experimental inquiry in science. Where Steinle drew on the example of $19^{\text {th }}$-century electromagnetism to characterize exploratory experimentation as a research activity driven by "the elementary desire to obtain empirical regularities and to find out proper concepts and classifications by means of which those regularities can be formulated" (Steinle, 1997, p. S70), Burian cited work in mid-20 $0^{\text {th }}$ century histochemistry as aiming for a stabilization of phenomena, notably via "stabilization of the protocols for locating particular molecular species" and for rendering them "relevant to the experimental and theoretical analyses of such other investigative traditions" (Burian, 1997, p. 42) as may be available. Exploratory experimentation, as Steinle puts it, cannot be divorced from "the process of forming and stabilizing the [new] conceptual framework" (Steinle, 1997, p. S72), which - needless to say - is made all the more difficult by the fact that, typically, in exploratory sciences "no wellformed theory or even no conceptual framework is available or regarded as reliable" (Steinle, 1997, p. S70). C. Kenneth Waters gives a rather succinct, yet insightful characterization of the relation between exploration and the absence (or at least inaccessibility) of fundamental theory, when he writes that "the aim of exploratory experiments is to generate significant findings about phenomena without appealing to a theory about these phenomena for the purpose of focusing experimental attention on a limited range of possible findings" (Waters, 2007, p. 279) (italics original).

As this brief characterization makes plain, exploratory experimentation cannot easily be - and certainly should not be! - assimilated to a view of science that regards experiments as exclusively, or even just primarily, in the business of testing theoretical hypotheses. For one, as the various discussants cited above agree, no well-formed body of theoretical principles from which such hypotheses may be derived exists in exploratory settings, and sometimes even the conceptual frameworks themselves are part of what is at issue. Testing, then, can at best be a tentative and local affair, and will necessarily be heavily invested with contentious background assumptions, since it will not generally be the case that one can simply hold the "theoretical" part of one's exploratory investigations fixed. This is why, as Kevin Elliott puts it, exploratory experimentation is perhaps best understood as "an attempt to study a phenomenon using as many tools and techniques as possible so as to understand it more fully and to gain more solid epistemic access to it" (Elliott, 2007, p. 328). Necessarily, this process of getting a grasp on a phenomenon will often require multiple attempts and approaches from different directions. Hans-Jörg Rheinberger captures the spirit of such exploratory inquiry well, albeit in somewhat metaphorical language, when he insists that an "experiment is not only a test or an examination", but more frequently "is an exploratory movement, a game in which one plays with possible positions, an open arrangement" (Rheinberger, 2010, p. 247). However, unlike in a well-defined game, where the rules specify when the game is over and who the winner is, in exploratory settings there is no guarantee that, at the end of an episode of inquiry, we will find ourselves having obtained a "winning" (true) hypothesis - even if exploration is carried out in the hope of at least identifying a fruitful approach. This reflects the hope, long cultivated by proponents of the heuristic value of abductive reasoning in science, that in contexts where it is unclear what is required in order to show a hypothesis $H$ to be true (or false), we may yet be able to gain a better sense of 
"what is required for deciding that $H$ constitutes a plausible kind of conjecture" (Hanson, 1960, p. 93).

At the same time, it would be misleading to think of exploration as devoid of all theory. Theory can enter at various levels, sometimes explicitly so, without thereby reducing exploratory experimentation to merely a means of 'testing' theoretical hypotheses. For one, significant background knowledge - including background theory - will often be required for devising experiments if the latter are to improve our understanding of the phenomena that stand in need of 'stabilization'. Furthermore, interpreting data and comparing experimental designs will likewise often be informed by theoretical considerations, even when the latter do not drive interpretation and design. Finally, as Elliott has noted, theory often "plays the role of a starting point of a 'foil' in the exploratory process" (Elliott, 2007, p. 327); this observation is borne out by a growing number of historical case studies of exploratory experimentation across the physical and biological sciences. Thus, while exploratory research often proceeds in the absence of a fully developed theory, is not driven by pre-existing theoretical concerns, and does not primarily aim at testing theoretical predictions, it need not (and perhaps could not) be entirely theory-free either.

In order to avoid possible misunderstanding, it is perhaps worth drawing two further contrasts, with abduction and heuristics, respectively. Though closely related to explorationinsofar as both notions also aim to shed light on the interplay between discovery and justification - they highlight somewhat different aspects of the process of scientific inquiry and innovation. In its most generic form, abduction may be described as the process of inferring hypotheses (purported facts or, in science, laws of nature) that, if true, would render certain sentences plausible. Thus, if the latter report observations that have been made, abduction is of explanatory use - the inferred hypotheses are (potential) explanations of what has been observed - whereas if they concern predictions, it may be a powerful tool for unearthing new discoveries. Though the term 'abduction' is sometimes reserved for the process of generating plausible hypotheses (irrespective of their relative merits), more often than not it is equated to the process of inference to the best explanation, which has an obvious evaluative component. 'Abduction', then, may refer both to the generation of hypotheses concerning a given empirical phenomenon and to the process of assessing their relative merit. Yet note that both of these established usages take the existence of the explanandum for granted: what is at issue is not whether a stable phenomenon exists, but what explains it. By contrast, in contexts of exploratory research, it is often an open question whether or not a series of observations exhibits sufficient unity to count as a stable research object in the first place; exploration, in such a situation, may consist in probing the (experimental and/or theoretical) conditions under which one can hope to zero in on a purported phenomenon. In this sense, exploration may very well precede any explicit attempt at abductive inference. ${ }^{3}$ Regarding the second contrast, one first needs to distinguish between the use of heuristics as mental shortcuts in judgment and decision-making - which, as a fact about human psychology, can be expected to play an important role in exploration as much as in any other domain of human cognition and behaviour - and the heuristic appraisal specific to science,

\footnotetext{
${ }^{3}$ Lorenzo Magnani has proposed the notion of "manipulative abduction" (Magnani, 2004), which appears to have greater affinity to exploration as discussed in this chapter than the standard "theoretical abduction"; on this point see also (Gelfert, 2016, pp. 116, fn. 6).
} 
which "evaluates the promise, the future potential (including what is at stake), the problemsolving capacity, or what we might call the 'opportunity profile' of a claim, technique, proposal, etc." (Nickles, 2006, p. 161). To the extent that both may feature in science, they are concerned with (and are acknowledged by their proponents to be a matter of) "considerations relating to economy of research" (Nickles, 2006, p. 159) and to the efficient allocation of (material or temporal) resources. As such, they figure in "all stages of research" (Nickles, 2006, p. 165), not just in exploratory research. Though heuristic appraisal may encourage researchers to pursue exploratory strategies, as doing so may - at various points in scientific inquiry - be deemed the most promising allocation of resources, it is not itself identical to exploration. Exploration, in a nutshell, cannot be easily assimilated to either abductive inference or heuristic appraisal.

\section{Functions and uses of exploratory models}

While exploration precedes explicit hypothesis-testing and the emergence of a settled theory of the phenomena in question, it need not - as the discussion in the previous section makes plain - be shielded from rational analysis. Even as the criteria for what constitutes fruitfulness may vary across subdisciplines, agreement as to whether a given exploratory move has been more or less fruitful can often be reached, sometimes quite straightforwardly. Why is this so? In the case of exploratory experimentation, a strong case can be made that in spite of its independence from specific theoretical frameworks regarding the target phenomenon, "experimental activity may well be highly systematic and driven by typical guidelines". Such guidelines, as Steinle puts it, are of a "general methodological type" (Steinle, 1997, p. S70). At a descriptive level, they will be instantiated by recurring exploratory strategies; that is, by typical moves an experimenter can make in an attempt to gain an epistemic foothold. Some of these may seem trivial - an experimenter might, for example, systematically vary the different experimental parameters to see which changes have an influence on the phenomenon under investigation - others may require considerable ingenuity, such as trying to isolate hidden factors in order to find out which are superfluous and which are indispensable. Crucially, which exploratory strategies are promising, and which constraints they are operating under, will depend on the type of inquiry - e.g., measuring, experimentation, modelling, computer simulation etc. - and on the particulars of a given case. For example, when performing a field experiment in ecology, isolating a putative causal factor, or varying experimental parameters, will arguably take great skill and require significant resources; by contrast, when numerically modelling a phenomenon using a polynomial equation, systematically varying the model parameters may border on the trivial. As in the case of experimentation, then, exploratory uses of models need to constantly (or at least regularly) be assessed for their fruitfulness and suitability to the task at hand.

A close affinity between modelling and exploration is only to be expected, given the origins of scientific models in what, at the time, were called 'mechanical analogies'. When, for example, James Clerk Maxwell set out to get a grasp of electromagnetic phenomena, he proposed his molecular vortex model which, in retrospect, may be characterized as an attempt to model the electromagnetic field in mechanical terms. Maxwell is explicit about the 
exploratory role of his vortex model: anyone who understands its "provisional and temporary character", he argues, "will find himself rather helped than hindered by it in his search after the true interpretation of the phenomena" (Maxwell, 1890, p. 486). That his model was not merely a prop for the theoretical imagination, in the way that a sketch of a geometrical figure might be for a mathematician trying to construct a proof, is evident from the fact that Maxwell frequently moved back and forth between viewing the model as auxiliary and crediting it with new ontological content which allows it to guide our inquiry into the nature of electromagnetic phenomena. (See, e.g., (Harman, 1998).) In such cases, to adapt a passage written by Herman Meyer as early as 1951, "the construction of scientific models" itself is an instance of our "specifically human way of progressing from phenomenological description to scientific knowledge"; it is, Meyer agues, "a mental operation sui generis" (Meyer, 1951, p. 118). As we shall see, this point - that the construction of a model, irrespective of its detailed empirical performance, can afford knowledge - can be made more precise by highlighting the role of exploratory models in constructing what one might call proofs of principle.

Before turning to a list of some of the distinct functions that exploratory models can serve, it is worth highlighting just one of the many strands in philosophical theorizing about what models are. (For a review of the ontology of models, see (Gelfert, 2017).) The starting point for this way of thinking about scientific models is somewhat akin to Meyer's characterization of model construction as a "mental operation" in its own right, in that it ties scientific models closely to the cognitive processes involved in mental modelling. The recognition that much of human reasoning proceeds with 'mental models', by carrying out thought experiments on internal representations of scenarios (rather than by rigorously applying logical rules to propositional representations), has led some philosophers of scientific models, for example Nancy Nersessian, to the view that a mental model is "a structural analog of a real-world or imaginary situation, event, or process", where this is meant to convey that "it embodies a representation of the spatial and temporal relations among, and the causal structures connecting the events and entities depicted and whatever other information that is relevant to the problem-solving tasks" (Nersessian, 1999, p. 11). There is, on this view, considerable continuity between the way we construct scientific models and the way we represent the world around us in thinking, whether we actively seek to represent real-world situations or, as in the quote above, imaginary situations, events, or processes. Given this symmetry between representing real and imagined, actual and possible scenarios, it should not come as a surprise that models - including, importantly, scientific models - allow us to extrapolate beyond the actual, thereby allowing us to also explore possible, e.g. counterfactual, scenarios. The use of models, then, is not restricted to the initial goal of representing actual target systems. Indeed, some classes of scientific models - such as toy models - typically only aim to provide potential explanations of general patterns, which happen to be actually instantiated, without thereby claiming to be able to identify the actually operative causal or explanatory factors. ${ }^{4}$ For present purposes, I shall neither pursue different accounts of what, in general, scientific models are, nor limit myself to just one class of models; rather, I shall focus on some important functions of exploratory modelling, giving

\footnotetext{
${ }^{4}$ On this point, see (Reutlinger, et al., forthcoming).
} 
brief examples of each, before turning to an in-depth example, of models of biological pattern formation, in the next section.

It is important to preface the subsequent discussion with a disclaimer to the effect that any list of exploratory functions of scientific models is likely to be incomplete and tentative. In distinguishing between four general functions of model-based exploration, I am following recent analyses by emphasizing that these functions are neither exhaustive nor mutually exclusive. ${ }^{5}$ Indeed, as we shall find, the same model may sometimes serve different exploratory goals at the same time. Another disclaimer concerns the fact that the models discussed here, for the most part, are formulated in the language of mathematics. While this is not by chance, it should not be taken to imply that exploration, in any of the senses to be discussed, is restricted to mathematical models only. A full explanation of why mathematical models lend themselves so well to exploratory uses is beyond the scope of this paper and would require close engagement with the literature concerning the applicability of mathematics to the natural sciences and with the role of well-developed symbolic formalisms in guiding, and constraining, the making of inferences. ${ }^{6}$ For present purposes, and in order to defuse worries about the choice of examples, perhaps it will suffice to note that scientists themselves - including those from the non-exact sciences - frequently presuppose this connection indirectly, and sometimes note it quite explicitly. Thus, in one of the rare explicit discussions of the exploratory role of models, the ecologist Peter J. Taylor notes that, while many theoretical and conceptual tools in biology function as schemas, i.e. as way of highlighting relevant basic processes, constraints, or (e.g. reproductive) strategies, "if the schema can be expressed in a mathematical formulation, the model becomes what I call an exploratory tool'. Mathematical investigation, Taylor notes, in a manner reminiscent of Steinle's exploratory strategy of varying experimental parameters, allows biologists to study how "the system's behaviour changes as its parameters change or becomes variables, as time lags are added, and so on" (Taylor, 1989, p. 122). In doing so, it allows for the formulation of new research questions or may suggest new terms and classifications, e.g. of systems-level behaviours.

In distinguishing between different exploratory functions of models, it makes sense to proceed from 'weaker' to 'stronger' versions, where this does not imply that any such ordering is either necessary or unique. Rather, for reasons of exposition, it will be helpful to realize that there is a spectrum of exploratory strategies, some of which are more ambitious than others. At the 'weak' end of the spectrum, then, we find exploration being conducted in the hope of identifying fruitful ways of proceeding, in spite of the absence of a well-formed underlying theory. As William Wimsatt notes in relation to scientific models, an "oversimplified model may act as a starting point in a series of models of increasing complexity and realism" and may serve "to generate new predictive tests of or to give new significance to features of an alternative preferred model” (Wimsatt, 2007, p. 104/127). This first, relatively weak function of exploratory modelling may thus be described as aiming at starting points for future inquiry. One might worry that, in the early stages of research, virtually any move by a scientist or modeller, in some shape or form, aims at enabling future

\footnotetext{
${ }^{5}$ On this point, see also (Gelfert, 2016, p. 95).

${ }^{6} \mathrm{I}$ discuss these issues in more detail in (Gelfert, 2016), (Gelfert, 2014), (Gelfert, 2011), and (Gelfert, 2009).
} 
inquiry, so that - in the absence of additional criteria for what should count as exploration even "back-of-the-envelope" sketches would count as 'exploratory models' in this first sense. This may be so, but simply means that we may need to exercise a certain amount of selfrestraint in deploying the label. History and context will usually allow us to distinguish quite clearly between mere uninformed guesswork and fruitful, informed exploration that led to increasingly realistic and sophisticated models. Consider the case of models of traffic flow in sociodynamics. Early such models, which looked towards fluid dynamics for inspiration, were unsuccessful at explaining various features of the flow of human-operated vehicles. By the middle of the $20^{\text {th }}$ century, it had become clear that, for a model of car traffic to have any chance of being successful, it would need to include a variety of disparate influences, from physical quantities (such as velocity, acceleration, geometry of the vehicles) to psychological phenomena (e.g., reaction time of drivers). In 1953, the American engineer Louis Pipes proposed the first car-following model (Pipes, 1953), which was based on the idea that traffic flow is nothing but the cumulative effect of each driver, $n$, responding to the car in front of her, $n-1$. The corresponding positions can then be modelled, with $d$ being the distance between the two vehicles at rest, $l_{n-1}^{v e h}$ the length of the car in front, and $T v_{n}$ the (velocitydependent) 'legal distance', in the form of the following equation:

$$
x_{n-1}=x_{n}+d+T v_{n}+l_{n-1}^{v e h} \text {. }
$$

It is clear that this equation is neither fundamental nor complete, but - as the subsequent proliferation of more refined car-following models demonstrates (see (Wageningen-Kessels, et al., 2015)) - it proved a fruitful starting point for thinking of traffic as the cumulative effect of how drivers respond to each other.

The second and third functions of exploratory modelling I wish to highlight - viz., providing proofs of principle and potential explanations - can, without much loss in terms of the sharpness of this distinction, be illustrated using the same example from population biology, the Lotka-Volterra model of predator-prey dynamics. This model has become the textbook explanation for one of the most intriguing phenomena in population biology, namely the correlated oscillations in the size of populations of predator and prey species. Mathematically, the model consists of a pair of first-order, non-linear, differential equations linking the population size of one species to that of the other, in such a way as to reflect that one species is feeding on the other. The rate of change in each population is thus dependent on the other, but not in exactly the same way. Since the prey is typically a fast-reproducing species, its population size is primarily determined by reproduction (proportionate to the existing population size) and mortality due to predation (proportionate to its own population size and to that of the predator species). By contrast, the predator species is modelled as having a constant death rate, so the total number of deaths is proportionate to its population size, while its population growth (=the total number of births) is assumed to be proportionate to both its own population size and to that of the prey which, after all, sustains the predator species. Mathematically, this is represented as follows (with $x$ indicating the size of the prey population, $y$ the number of predators, $t$ time, $a$ the prey's birth rate, $b$ the predator's death rate, and $\alpha, \beta$ positive coefficients representing the effect each population has on the other):

$$
\frac{d x}{d t}=x(a-\alpha y)
$$




$$
\frac{d y}{d t}=y(\beta x-b)
$$

Because of the way the variables representing the two population sizes depend on each other, they exhibit a remarkable behaviour in time: both populations will oscillate indefinitely, the predator population lagging behind slightly, while the prey population overshoots quite dramatically. (See fig. 1) No stable equilibrium exists, only an unstable one that could not withstand the slightest (and, in any real-world situation, inevitable) perturbation. In other words, the Lotka-Volterra model exhibitis a distinctive, cyclical pattern: When the number of predators is low, the prey population will increase rapidly, even as the predator population begins to recover, which in turn will grow and will eventually bring down the total number of prey below the number needed to sustain the (now increased) predator population.

\section{Population Size}

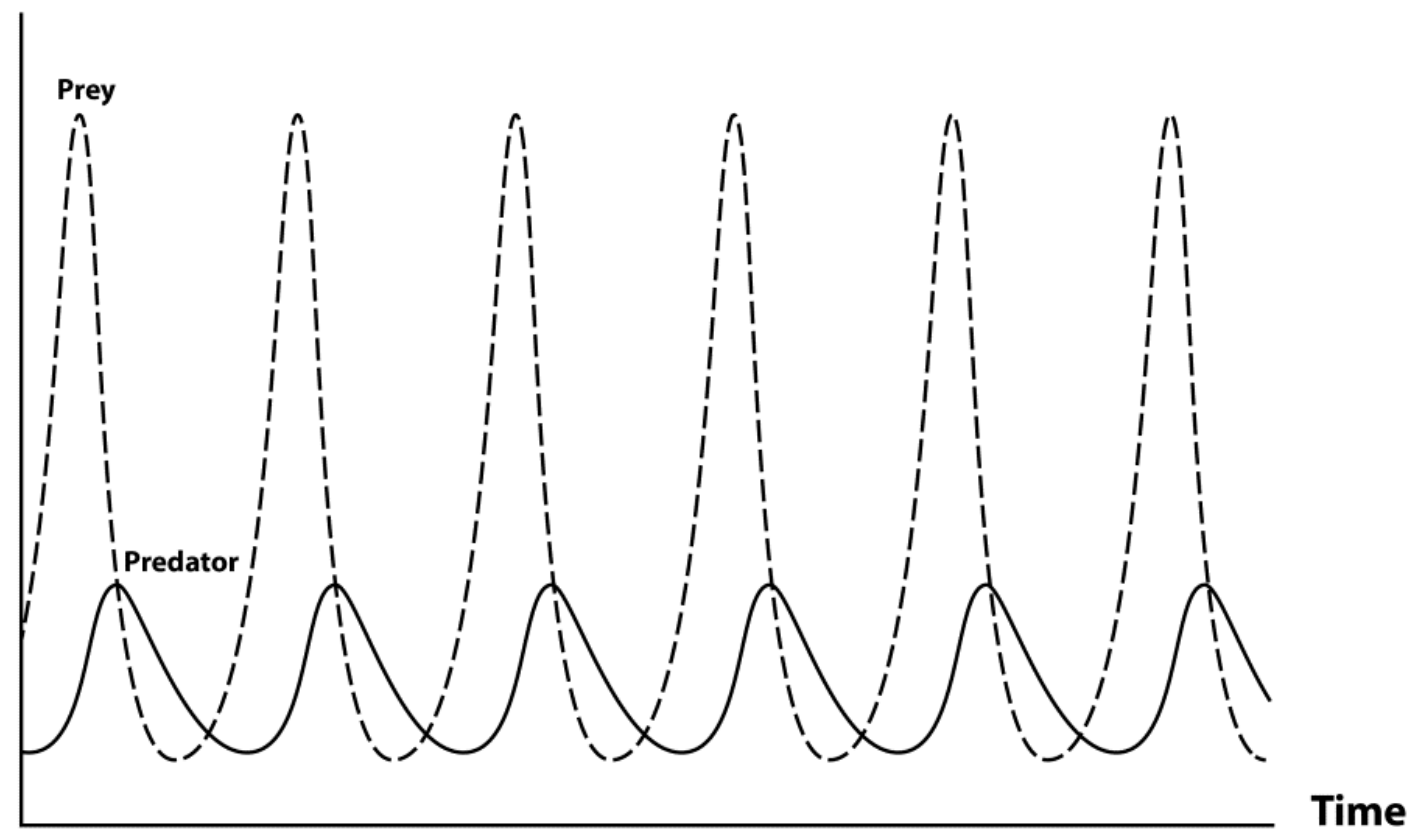

Figure 1. Lotka-Volterra model

What makes the Lotka-Volterra model significant as a tool of exploration is its dual character as a methodological proof of principle and a potential explanation. Consider the methodological aspect first. At any given point in time, the size of a population will be an integer number: there may be forty-five lynx roaming the forest, or four hundred twenty-two, but not 1.33 or $\sqrt{2}$. Changes in population size, likewise, come in units of one animal at a time: a live birth adds a member (or perhaps several) to the population, each death brings down the total number by one. Furthermore, births and deaths are discrete events in time, which raises the question of whether one should perhaps model population dynamics using discrete time steps. Yet, the Lotka-Volterra model demonstrates that it $i s$ possible to reproduce the qualitative behaviour of predator-prey systems using continuous differential 
equations; that is, it constitutes a proof of principle that the methodology of continuous differential equations is suitable for generating insight into the dynamics of (discrete) populations. But the Lotka-Volterra does more than prove the point that we do not always need a discretized model to make sense of how populations behave, it also offers a potential explanation of an empirically salient phenomenon: the cyclical patterns of population growth, followed by near-collapse, in predator-prey systems. These patterns, observed across various species in different ecosystems - e.g., in lynx and snowshoe hares in the Hudson's Bay area (inferred from hunting data) as well as in sharks and their prey (as reflected in the catch of fishermen in the Adriatic Sea) - had long been regarded as puzzling and as standing in need of an explanation. The Lotka-Volterra model explains how it is possible that such stark cyclical patterns, of rapid population growth periodically followed by steep decline, can emerge spontaneously, from within the two-species system containing the predator and prey species, without the need to posit any external forcings such as famine or disease.

A perhaps less obvious, but nonetheless important function of exploratory modelling is the search for, or revision of, potential target systems. It is common to think of models as tools for representing actual target systems, or indeed as "inherently intended for specific phenomena" (Suárez, 1999, p. 75). From this perspective it may seem puzzling why, when faced with a mismatch between model and target, we should ever consider reassessing the target system, rather than revising our model to better fit the target. But recall that in an ongoing episode of exploratory research, it is often not obvious whether one has even succeeded in properly delineating a stable target system. And even if one has, one may find that the model that resulted from aiming at a specific target turns out to be better suited to the study of neighbouring phenomenon, or that one's model has unexpected applications outside the originally intended domain of phenomena. In other words, what the target of our modelling efforts will eventually be - or, in more extreme cases, whether we can even expect there to be actual target systems that fit - may well remain an open question for as long as exploratory research has not yet become settled science. ${ }^{7}$ Sometimes, as the title of this chapter suggests, our models are in search of targets, and the next section will develop in more detail one such example of a well-developed set of model equations whose overall applicability to real target systems remains very much a matter of dispute.

\section{The case of reaction-diffusion models for biological pattern formation}

Only a couple of years before his untimely death, Alan Turing published an ambitious article, "The Chemical Basis of Morphogenesis" (Turing, 1952), which set out "to account for the main phenomena of morphogenesis" by providing "a possible mechanism by which the genes of a zygote may determine the anatomical structure of the resulting organism". Turing was careful to emphasize that he did not wish to "make any new hypotheses" of a biologically

\footnotetext{
${ }^{7}$ It is also important to keep in mind that there is value in modelling counterfactual situations, including scenarios that could not possibly be instantiated in the actual world (such as higher-dimensional systems in physics).
} 
substantive kind; rather, he aimed to demonstrate in general terms how it may be the case "that certain well-known physical laws are sufficient to account for many of the facts" of the development of biological form. Previous attempts to apply mathematics to the problem of biological form, notably D'Arcy Thompson's influential treatise On Growth and Form (1917), essentially tried to recreate various actual biological shapes in mathematical form and, via the method of transformed coordinates, showed how they can be transformed into one another. (Thompson, 1917) Yet, while Thompson's text contains considerable discussion of the constraints on biological forms - arising from differences in the relative importance of the various forces, like gravitation and capillary forces, all operating at different scales overall there is little concern with modelling potential causal mechanisms. By contrast, Turing explores biological pattern formation by mathematically modelling a possible mechanism how biological form develops from the zygote.

Turing's starting point is a general puzzle concerning the origins of biological form. All organisms begin as undifferentiated zygotes that, for all intents and purposes, exhibit no preferred directionality or spatial structure. On the one hand, an embryo in its early (blastula) stage, exhibits "spherical symmetry, or if there are any deviations from perfect symmetry, they cannot be regarded as of any particular importance, for the deviations vary greatly from embryo to embryo within a species, though the organisms developed from them are barely distinguishable" (Turing, 1952, p. 41). On the other hand, fully grown organisms, such as a horse ("which is not spherically symmetrical", as Turing quite rightly notes; ibid.), have considerable internal structure, which defines their morphological characteristics. It might seem puzzling how, at some point during an organism's development, the symmetry and homogeneity of the early embryo can give way to distinctive, and formative, spatial patterns. Part of the puzzlement regarding this transition, however, arises from what Turing calls the "fallacy" of assuming "that the deviations from spherical symmetry in the blastula could be ignored because it makes no particular difference what form of asymmetry there is" (Turing, 1952, p. 42). For, it is important that some such deviations will inevitably occur, and if a developing embryo responds by amplifying them in systematic ways, it may reach a new and stable equilibrium that lacks the original symmetry and brings determinate spatial patterns into existence. In his paper, Turing aims to specify just such a mechanism, by which small fluctuations can trigger, in a predictable and systematic fashion, pattern formation in what would otherwise be homogeneous systems.

The central idea behind Turing's model is that two (or more) diffusible substances (dubbed "morphogens") can interact with each other in such a way as to form "reactiondiffusion systems" which establish chemical gradients within an organism, which, in turn, give rise to self-organized biological patterns. For such pattern formation to emerge spontaneously in an otherwise homogeneous system, at minimum two linked morphogens are required. If only one morphogen were present, though it could be locally produced and diffuse across an organism, it could at best trigger the expression of a preformed pattern, e.g. by "switching on" a gene that was unevenly distributed across the organism (or responded to different concentrations of the same morphogen). It could not, however, bring about, say, periodic patterns in an otherwise homogeneous population of cells. Adding a second morphogen changes the situation fundamentally, provided we allow for the possibility of the rates of production and activation of the two substances being coupled to one another. In 
addition to diffusion, we then have a reaction that produces the morphogens, in ways that are not merely superpositions of two independent processes, but are dynamically coupled.

Turing's model, thus, includes two morphogens, $S$ and $P$, diffusing homogeneously (except for random fluctuations) within a certain space, with one being locally self-enhanced and the other being capable of long-range inhibition of the first. Because of the differential diffusion rates of the two molecules, and the way in which the more slowly diffusing molecule both stimulates the production of itself (self-enhancement) and the production of its own inhibitor, a range of possibilities for pattern formation opens up. To see how this may occur, consider a random distribution of the two morphogens at time $t_{1}$ and its evolution over time (see fig. 2).

Time 1

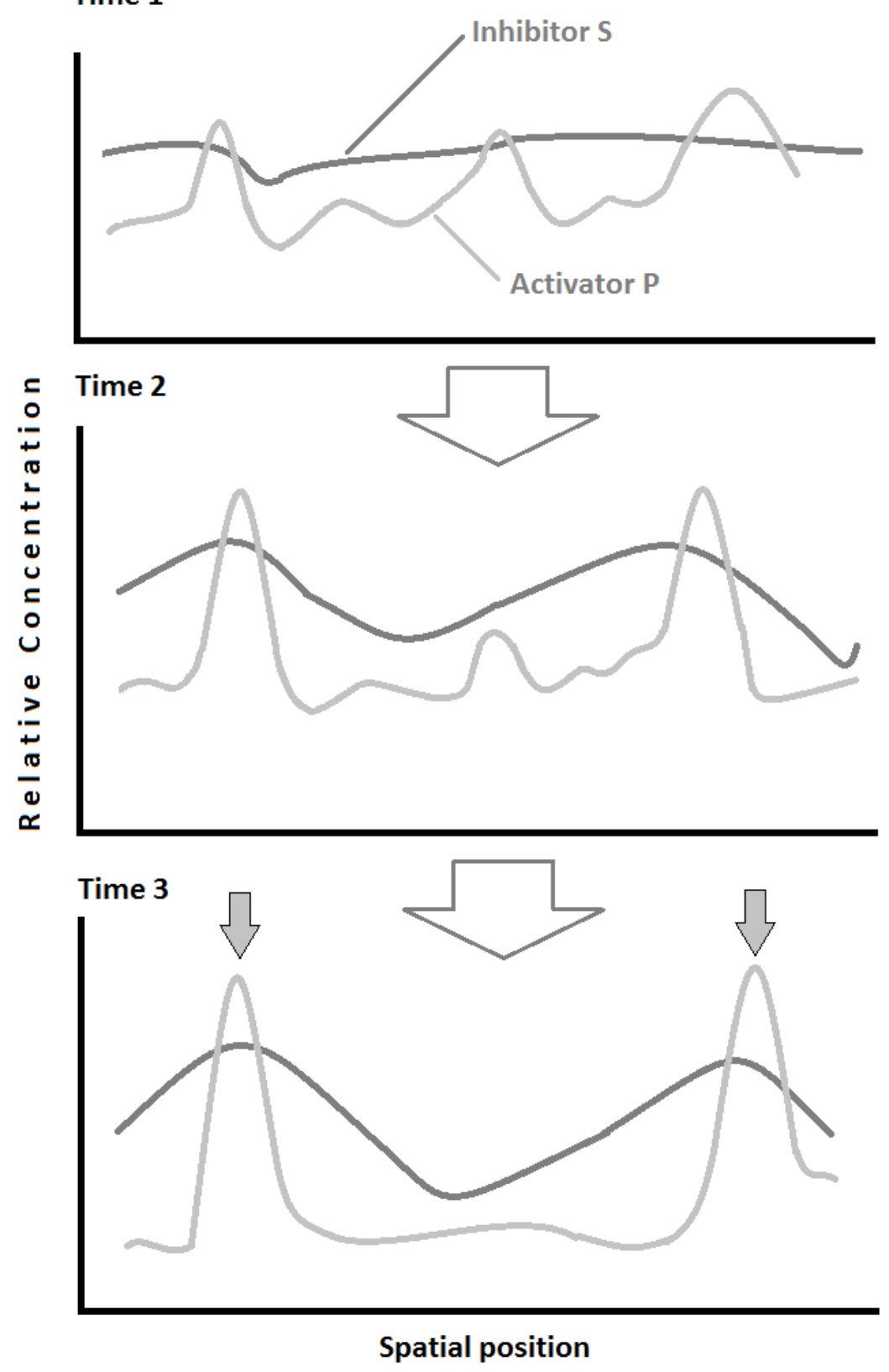

Figure 2. Reaction-diffusion time evolution 
$P$ enhances its own production (e.g. autocalytically) and diffuses slowly, so has a tendency to concentrate into peaks, even without the presence of $S$ (time $t_{1}$ ). Moreover, since $P$ also stimulates production of $S$, its own (quickly diffusing) inhibitor, these peaks will become more localized as $P$ 's concentration will fall quickly as one moves away from a given peak (time $t_{2}$ ). Of course, as $S$ diffuses further in space, its concentration will eventually fall below the level needed to inhibit the formation of further peaks in the concentration of $P$. Where $S$ is sufficiently diluted, new peaks of $P$ can emerge in the same fashion. The result is a "standing wave" pattern, with localized peaks of $P$ at more or less regular intervals in space (time $t_{3}$ ).

The discussion so far makes it plausible that the mutual interaction between two substances, a (self-)activating morphogen and a quickly diffusing inhibitor which together have the twin effects of local activation and long-range inhibition, can explain spontanenous pattern formation in what would otherwise, barring inevitable fluctuations, be a homogeneous medium. The specific pattern, of course, will depend on the various parameters that characterize the reaction and diffusion process: the rate of production of each substance (and its dependence on the concentration of the other), the rate of degradation and the differential speed with which each substance diffuses. Mathematically, this is captured by the following set of equations, which express the rates of concentration change as the sum of reaction terms for the production, degradation, and diffusion of the two morphogens, respectively:

$$
\begin{aligned}
& \frac{\partial u}{\partial t}=F(u, v)-d_{u} v+D_{u} \Delta u \\
& \frac{\partial v}{\partial t}=G(u, v)-d_{v} v+D_{v} \Delta v
\end{aligned}
$$

where $u$ and $v$ refer to the concentrations of the $P$ and $S$, respectively. Not all mathematically stable solutions to the model equations are associated with the formation of salient and stationary patterns; some give rise to oscillatory waves or uniform distributions. Those solutions that essentially correspond to stationary waves with finite wavelength - or, Turing patterns, as they are now called - themselves exhibit great variety (see fig. 3). Visual inspection shows that these basic solutions already bear significant resemblance to actual patterns found in various organisms, such as giraffes, the striped zebrafish, and the African leopard. Through minor modifications - e.g. by tweaking the boundary conditions, or 'adding on' another Turing-style system of morphogens - it is possible to recreate a vast number of different arrangements of spots, stripes, swirls, and splodges, such that, through clever choice of parameters, it should be possible to explain various types of patterns found in nature. Or so it would seem. 

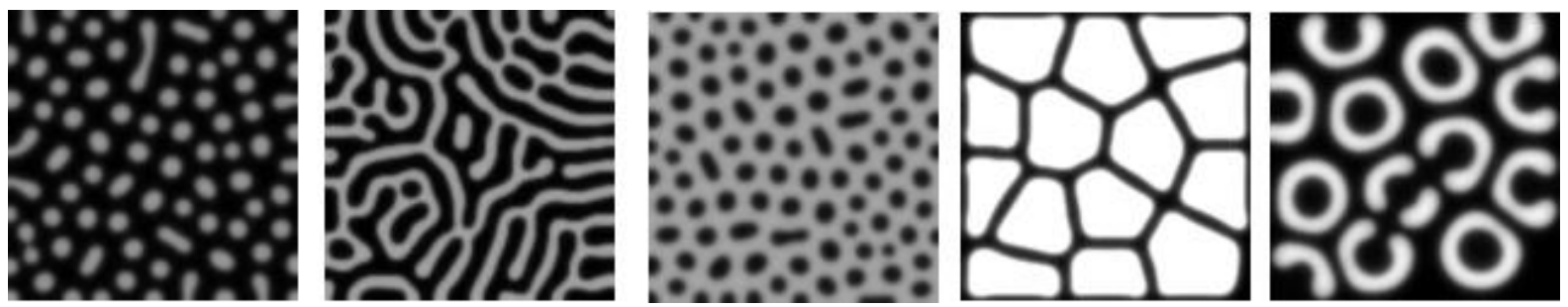

Figure 3. Turing patterns. (Image courtesy of Shigeru Kondo.)

Turing wrote as a non-biologist at a time when little was known about the molecular basis of biological pattern formation. So it is hardly surprising that he was unable to specify any actual biological examples of his two-morphogen reaction-diffusion model. Neither was this his goal: as he makes clear throughout the paper, his main objective - in line with our third function of exploratory modelling discussed in the previous section - was to provide a potential explanation of how biological patterns form spontaneously as part of an organism's development. He is explicit in characterizing his model as merely "a possible mechanism" (Turing, 1952, p. 37) and, in the concluding paragraph of his paper, weakens this goal further when he expresses his hope "that the imaginary biological systems which have been treated, and the principles which have been discussed, should be of some help in interpreting real biological forms" (Turing, 1952, p. 72). Arguably, more is required than merely functioning as an interpretative tool if a model is to count as doing actual explanatory work. As Shigeru Kondo and Takashi Miura put it in a favourable review of half a century or so of work on the Turing model: "No matter how vividly or faithfully a mathematical simulation might replicate an actual biological pattern, this alone does not constitute proof that the simulated state reflects the reality." (Kondo \& Miura, 2010, p. 1617) It will be instructive, then, to look in a little more detail at the track record of Turing's model and how it has fared with respect to real-world cases.

Turing is not entirely silent on what kinds of pattern formation he thinks may potentially be explained by his model. First, he begins his paper by stating that he will describe "a mathematical model of the growing embryo" (Turing, 1952, p. 37), thereby suggesting that one of the explananda he is interested is how the early embryo's spherical symmetry is broken and how organisms acquire basic spatial organization. In this sense, Turing's professed goal is more ambitious than simply providing a potential mechanism for how certain surface characteristics - such as animal coat patterns - may form. Second, towards the end of his paper, Turing refers to a number of botanical examples, such as the arrangement, number, and symmetries of whorls of leaves of certain plants such as Woodruff (Asperula odorata). (The choice of the latter example - leaves growing around a circular stem of a plant - may be partly driven by his mathematical choice of a ring-like structure as one of the boundary conditions for his mathematical model.) Both kinds of cases, through no fault of Turing's, turned out to be less than ideal as illustrations of his model. From the late 1970s onwards, one of the main model organisms for the study of general morphogenesis was the fruit fly (Drosophila melanogaster), largely due to its manageable size and quick generation time, which made fruit flies the preferred model of how spatial organization comes about. However, while early embryonic development in Drosophila does exhibit standing wave patterns that are reminiscent of what the Turing model might have predicted, 
further investigation showed development and spatial patterning to be far more complicated, with the sequence of segments in the fly's body being determined by a set of maternal, gap, pair-rule and segment polarity genes, each of which is responsible for a different aspect of the organism's orgniazation (e.g., anterior/posterior asymmetry, division into head, thorax, and abdominal regions, etc.). In other words, not only did the dominant model organism's morphogenetic development not lend itself to being modelled in line with Turing's proposal, the very complexity of the actual developmental process suggested that the simple design of the Turing model might simply not have been favoured by evolution.

The case of plant development may be seen as even less convincing. For one, plants, due to their fixed location in space, may be subject to external factors (such as the relative position to the sun) whose influence could, at least potentially, suffice to break the symmetry, which would render the example rather less relevant to the question of embryonic morphogenesis in (esp. higher) animals. For plant morphogenesis in general, there must likewise be other considerations and constraints, as Turing was well aware. Recall that stable Turing patterns may be thought of as 'standing waves' of sorts (i.e., patterns that are brought about by the 'standing wave'-like distribution of the relevant morphogens in the organism). Whorls of leaves are presumed, by Turing, to "originate in rings of active tissue" (Turing, 1952 , p. 68), with the number of leaves corresponding roughly to the circumference of the ring divided by the 'chemical wavelength' of whatever morphogen is responsible for leaf formation. Whereas in species such as Asperula odorata the arrangement of between five and nine leaves into a whorl fits reasonably well with Turing's idea, his model cannot easily be extended even to something as closely related as flower petals. For, as Turing himself notes, "when all species are taken into account one must expect that the diameters of the rings concerned will take on nearly all values within a considerable range" (Turing, 1952, p. 69) and that there will also be some variation in chemical wavelength. By the logic of his own model, then, the number of petals on flowers should be expected to vary considerably, less so within the same species (though even there we should expect the variation across individual specimens to give rise to significant numerical variation in the number of petals and other elements), but most definitely across different species since, in principle, within a certain reasonable interval, any number should be as likely as any other to be the result of dividing the circumference by the (likewise variable) chemical wavelength. Yet, as Turing notes, this is not what one finds in nature: "The number five is extremely common, and the number seven rather rare.” (Turing, 1952, p. 69)

For the longest time, developmental biologists regarded Turing's model as mathematically elegant, but biologically irrelevant. There are many reasons why this was so, and a full discussion of the history of the Turing model and its reception is beyond the scope of this paper. Suffice it to say that Turing's mathematical approach and his formal presentation, together with the underdeveloped (and ultimately unconvincing) biological examples he had given, did not mesh well with the epistemic culture of developmental biology. Solving the equations of Turing's model even approximately required mathematical training and ingenuity that few developmental biologists had, and by the time computer simulation methods were gradually becoming more widely used, another - more intuitive model, Lewis Wolpert's 'French flag model' (Wolpert, 1969), had won over the majority of those developmental biologists interested in biological pattern formation. 
In order to understand Wolpert's model, consider a layer of cells which, for ease of illustration, we can imagine to be in the shape of a rectangle. A group of cells along the lefthand edge of the tissue is continuously producing a single morphogen $S$ which diffuses linearly, so as to create a smooth gradient from left (high concentration of $S$ ) to the right (low concentration). (Let us further posit that, on the right-hand edge, there is a 'sink', where $S$ disappears, or perhaps decays, so that we need not worry about saturation due to the continuous production of $S$ on the left-hand side.) Once a stable distribution has been reached throughout the system, the concentration of $S$ at a given point in space effectively provides positional information regarding how far along the rectangular strip of tissue we are. In a second step, cells can then respond to the positional information that is available to them. In the simplest case, a cell might simply be programmed to react differently to different concentrations of $S$ around it. If, for example, cells reacted to high concentrations of $c>c_{B}$ (where $c_{B}$ is a threshold concentration required for certain genes to be 'switched on', say) by turning blue, to intermediate concentrations $c_{B}>c>c_{R}$ by turning white, and to low concentrations $c_{R}>c$ by turning red, what would emerge in the scenario described is a tricolor pattern, blue-white-red: hence, the designation of 'French flag model'. Empirical support came from a variety of experiments and observations. In a series of experiments in the late 1950s and early 1960s, researchers identified signal-producing structures of just the kind described in the thought experiment: in experiments with chicken embryos, it was found that the apical ectodermal ridge, a thick layer of ectoderm that rims the tip of the developing limb bud, is crucial to chondrogenesis. If removed, the formation of the limb would be truncated, whereas if a piece of thigh mesoderm was grafted on top of it, rather than developing into more thigh tissue, it would grow into (sometimes an additional) limb. (See (Saunders, et al., 1957).) Additional support for the idea that it only takes a single morphogen to 'unlock' different genetic programmes in response to the positional information contained in its concentration gradient comes from the further observation that, in many organisms (e.g., salamanders), whole limbs can be regenerated when removed. Furthermore, the French flag model can explain why patterns are stable even as overall size varies: if, as in the thought experiment, cells respond in only three distinct ways - by turning blue, white, or red - then, whether the area is small or large (and, hence, the concentration gradient more or less steep), one will always find a tri-color pattern. By contrast, a Turing mechanism would likely predict a variable number of stripes, due to the interplay between the chemical wavelength associated with the reaction-diffusion process and the overall geometry of the tissue sample. In light of such seemingly clearcut empirical evidence, and given the intuitive appeal of the 'French flag model', interest in the Turing model diminished. When, in 1979, Stuart Newman and Harry Frisch put forward a mathematical model of chick limb that had "affinities to that of Turing" (Newman \& Frisch, 1979, p. 662), this generated some initial interest, but did not as a whole diminish the dominance of the 'French flag model'. Though Newman and Frisch included a (given the computer technology at the time, highly 'pixelated') sketch of how chondrogenesis might come about over time within their model, this does not seem to have tipped the balance of evidence in their favour either - not least since the development of the chick's distinctive limb with its three, morphologically very different fingers was seen as an especially convincing case of how, in the spirit of the 'French flag model', very different patternings needed to be 'unlocked' for each of the three fingers. 
It was not until the mid-1990s that new experimental evidence and simulation techniques became available that led to a revival of interest in the Turing model. Importantly, it was the interplay between experimental manipulation and model-based simulation that, as we shall see, made all the difference. Recall that one of the perceived advantages of the 'French flag model' was its ability to explain how, in the words of a recent textbook, "the system could also regenerate the complete original pattern if it were cut in half" (Wolpert, et al., 2015, p. 29) or otherwise disturbed. In a series of experiments on striped tropical fish (Pomacanthus imperator, or Emperor angelfish), Shigeru Kondo and Rihito Asai (Kondo \& Asai, 1995) manipulated their distinctive striped pattern via laser ablation of pigment cells, which removed a small portion of a stripe. Instead of the parallel stripes simply regrowing to complete the original pattern, as would be expected from within the 'French flag model', the lower stripe changed direction and shifted upwards, partially completing the upper stripe, while maintaining the spatial interval between the two stripes. This led to a distinctive and reproducible pattern of regrowth, different from the original pattern, which was more in line with a dynamic response to the twin processes of local activation and long-range inhibition than with the expression of a preformed pattern or a simple 'triggering' of a response by a simple gradient. Computer simulations confirmed that a suitably adjusted Turing model, but not a 'French flag model', was able to account for the distinctive spatial patterning associated with regrowth. (See fig. 4; for a review, see (Kondo \& Miura, 2010).)
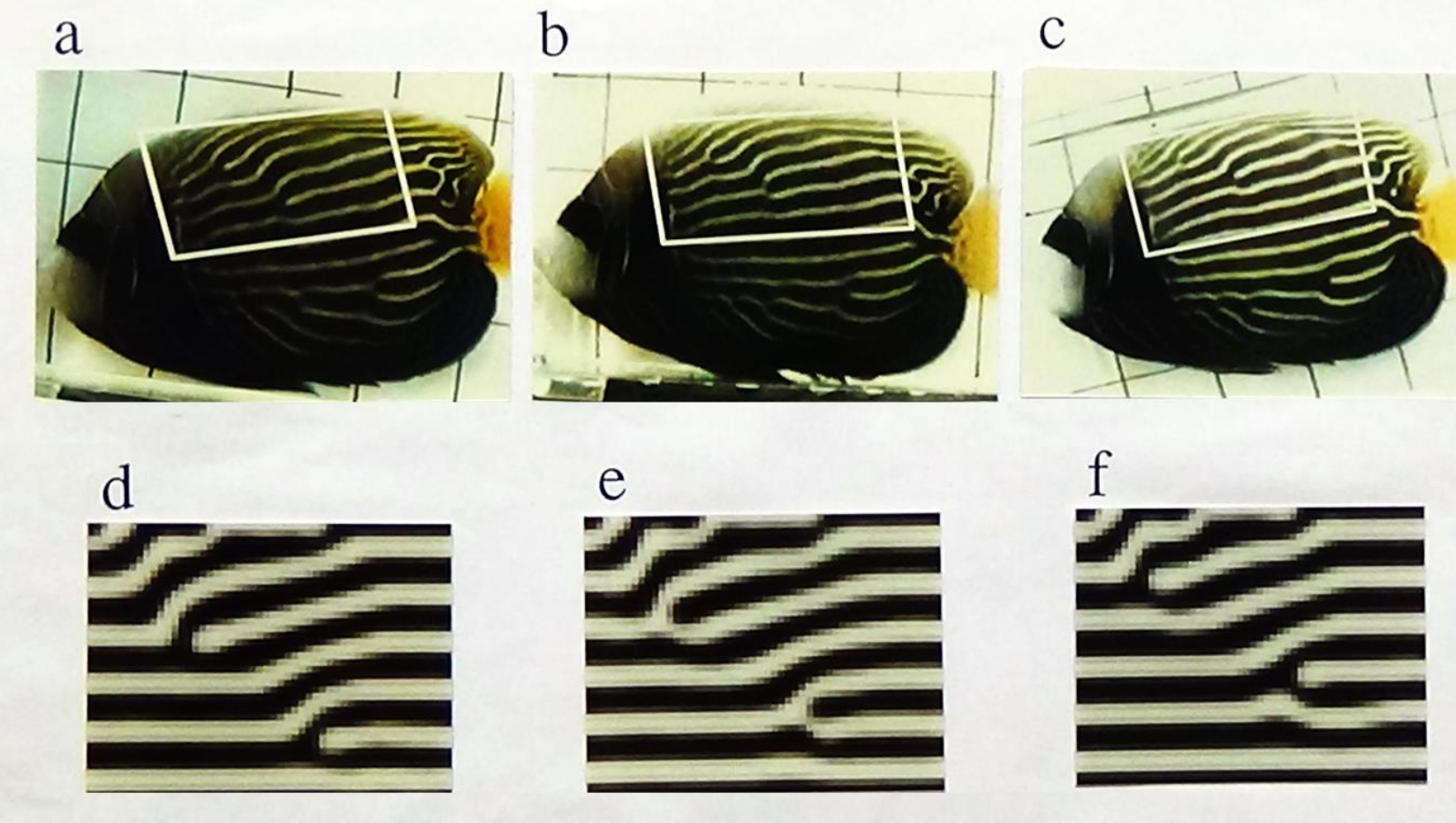

Figure 4. Re-arrangement of stripe pattern of Pomacanthus imperator; observation ( $a$-c) vs. Turing model simulations $(d-f)$. (Image courtesy of Shigeru Kondo.)

At least in part, then, it was due to the advent of better simulation techniques, which allowed for more subtle ways of exploring the phenomenon - for instance, by 'disturbing' (and otherwise manipulating) existing patterns and comparing the simulated response of the 
Turing system with observed patterns of re-growth - that Turing's approach gained new traction as a model of biological pattern formation. In addition, there has been the growing realization among researchers that, for the Turing model to be fruitful and afford insight into how biological pattern formation works, we need not actually discover two molecules whose chemical characteristics match those posited by Turing's account of the interaction between an activator and an inhibitor. Rather than treating Turing's model as a hypothetical representation of a two-morphogen system and making it our goal to discover an actual pair of substances that exhibit the posited chemical behaviour, we can consider the model as itself a hypothetical realization of the twin processes of local activation and long-range inhibition. After all, we should expect that what matters for self-organized pattern formation is not the precise number of different substances involved, or their specific chemical characteristics, but rather the fact that some underlying structure must realize the coupling of local activation with long-range inhibition. Interestingly, it was subsequently shown that in zebrafish the skin patterns are set up and maintained not directly in response to differential diffusion rates of two morphogens, but by a network of interactions between neighbouring pigment cells. While "the shape of the network is different from that of the original Turing model, it fits the short-range positive, long-range negative feedback description" (Kondo \& Miura, 2010, p. 1619). While it would thus be incorrect to consider the original Turing model a faithful representation of pattern formation in this particular species, it is in virtue of exploration on its basis that other realizations of local activation and long-range inhibition - in this and other species - were identified, and were found to be drivers of biological pattern formation of the same qualitative type as Turing had predicted. Indeed, in presenting their work, Kondo and his collaborators are adamant that they have identified "the essential conditions for generating the Turing pattern" in zebrafish and related species. (Pattern Formation Group, n.d.) By tweaking the original Turing model ever so slightly, it has thus become possible to open up ever more potential applications of the model. This has led to a reassessment of the original research agenda: no longer was the focus exclusively on embryonic development, but it has now come to encompass also the question of how biological organisms maintain the integrity of their morphological features, as demonstrated by Kondo and Asai's research on repair skin patterns in different fish species (see fig. 4). This nicely illustrates how exploratory modelling can reorient research agendas and can help identify productive new targets of scientific inquiry.

Contemporary developmental biology acknowledges the importance of the Turing mechanism as one of several key elements in the formation of biological patterns. While it may be too early, and while the field of developmental biology may be too diverse, to speak of a consensus, there appears to be a growing sense that real-life cases of pattern formation in biology often involve both, a (basic) periodic Turing pattern and a more specific patterning for each of the periodic elements thus brought about. A paradigmatic case would be limb formation. (See (Sheth, et al., 2012).) Whereas in the past it was thought that each digit was encoded by a separate genetic programme, research involving the 'knocking out' of Hox genes has convinced many researchers that, early on in its development, the limb bud is structured by a wave-like Turing pattern, and that the specific differences between digits are the result of further 'Wolpert-style' patterning of each individual element: in other words, the initial "periodic patterning is normally subject to a type of positional signal that varies along 
the proximodistal axis of the limb" (Green \& Sharpe, 2015, p. 1210). The story of the Turing model as presented in this paper, then, is not one of an "underdog theory" displacing "the received view": both models, Turing's reaction-diffusion model and Wolpert's positional information model, remain relevant and are legitimate and important sources of insight. Rather, what this historical episode illustrates is how a model that did, in a very clear sense, constitute a proof of principle for self-organized pattern formation, can go out of fashion and subsequently rebound. Some of the reasons that likely contributed to the initial lack of uptake among developmental biologists have already been mentioned: sociological reasons having to do with different epistemic styles (in this case, the clash between mathematical methods and experimentally-oriented developmental biology); lack of sufficiently sophisticated computer simulation techniques that would have allowed for a more detailed rendering of the finer details of Turing patterns (and which might have lent more credibility to Newman and Frisch's calculation of the development of the chick limb); and the absence of empirical observations of the posited two-morphogen systems. Yet, once this began to change, notably through the study of tell-tale distortions of small-scale structures in angelfish and zebrafish (thanks to the availability of new experimental and simulation techniques), the model was able to rebound - not necessarily as a faithful representation of pattern formation in any one species in particular, but as a tool of exploration, which allowed scientists to converge upon fruitful avenues of research. Indeed, the very fact that researchers now consider the Turing model useful not only in the (recently more promising; see (Maini, et al., 2006)) search for two-morphogen systems, but also in relation to other ways of realizing the twin processes of local activation and long-range inhibition - e.g., via networks of interaction between neighbouring cells - attests to the model's flexibility and exploratory potential.

\section{Conclusion}

At the beginning of this chapter, I wrote that exploration should stand alongside more traditional criteria for assessing models (such as explanatory, predictive, and representational success). The case of the Turing model illustrates why it may be worth valuing exploratory fruitfulness in its own right. At several crucial junctures in the Turing's model varied career, it would have been quite misguided to hold it to the standards of traditional hypothesistesting, given the level of generality at which it was formulated and the inability of scientists, at the time, to identify the molecular basis of biological development. To put things another way, had Turing's model been treated exclusively as a way of representing real target systems, it would have had to be regarded, if not as a non-starter, then certainly as empirically unsuccessful. And, as we have seen, such doubts about the model's applicability to real-world systems were common among developmental biologists. Yet, in spite of its perceived empirical shortcomings, the model was never discarded completely, and rightly so. This is because, in the spirit of Turing's own professed aim - that is, to offer no more and no less than "a possible mechanism" by which spontaneous pattern formation in living beings may occur - a minority of researchers recognized that the model's primary function was (and, to a large extent, remains) exploratory. That is, it was not geared towards representing any 
one target system, or class of target systems, in particular, but towards 'standing in' (or, as one might put it in order to highlight its function as a proof of principle, 'filling in') for whatever it is that realizes the twin processes of local activation and long-range inhibition and thereby brings about spontaneous pattern formation in some biological systems. From the start, the same basic model equations were intended to be potentially applicable to biochemically quite different potential target systems, and, as discussed in the previous section, this exploratory potential of the model was subsequently vindicated by novel observations across a range of species. Without an acknowledgment of the fundamental role of exploration in scientific modelling, the longevity of Turing's reaction-diffusion model which made its recent renaissance possible - would be little more than a historical anomaly, and its productive role in opening up new avenues of inquiry would have to be seen as a fortuitous, but ultimately puzzling accident.

\section{Acknowledgments}

I would like to thank the conference participants at the GWP.2016 meeting in Düsseldorf in March 2016 and at the workshop "Models in Science", held at Lingnan University (Hong Kong) in March 2017, for their discussion and feedback. An anonymous referee provided especially helpful and incisive comments, for which I am grateful. Professor Shigeru Kondo at Osaka University kindly gave permission to reproduce the two images labelled as figures 3 and 4 above.

\section{Bibliography}

Arabatzis, T., 2006. On the Inextricability of the Context of Discovery and the Context of Justification. In: J. Schickore \& F. Steinle, eds. Revisiting Discovery and Justification. Dordrecht: Springer, pp. 215-230.

Burian, R. M., 1997. Exploratory Experimentation and the Role of Histochemical Techniques in the Work of Jean Brachet, 1938-1952. History and Philosophy of the Life Sciences, 19(1), pp. 27-45.

Elliott, K. C., 2007. Varieties of Exploratory Experimentation in Nanotoxicology. History and Philosophy of the Life Sciences, 29(3), pp. 313-336.

Gelfert, A., 2009. Rigorous Results, Cross-Model Justification, and the Transfer of Empirical Warrant. Synthese, 169(3), pp. 497-519.

Gelfert, A., 2011. Mathematical Formalisms in Scientific Practice: From Denotation to Model-based Representation. Studies in History and Philosophy of Science, 42(2), pp. 272286. 
Gelfert, A., 2014. Applicability, Indispensability, and Underdetermination: Puzzling over Wigner's 'Unreasonable Effectiveness of Mathematics'. Science \& Education, 23(5), pp. $997-$ 1009.

Gelfert, A., 2016. How to Do Science With Models: A Philosophical Primer. New York: Springer.

Gelfert, A., 2017. The Ontology of Models. In: L. Magnani \& T. Bertolotti, eds. Springer Handbook of Model-Based Science. Heidelberg: Springer, pp. 5-23.

Green, J. B. \& Sharpe, J., 2015. Positional information and reaction-diffusion: two big ideas in developmental biology combine. Development, 142(7), pp. 1203-1211.

Hanson, N. R., 1960. Is There a Logic of Scientific Discovery?. Australasian Journal of Philosophy, 38(2), pp. 91-106.

Harman, P. M., 1998. The Natural Philosophy of James Clerk Maxwell. Cambridge: Cambridge University Press.

Hoyningen-Huene, P., 2006. Context of Discovery Versus Context of Justification and Thomas Kuhn. In: J. Schickore \& F. Steinle, eds. Revisiting Discovery and Justification. Dordrecht: Springer, pp. 119-131.

Kondo, S. \& Asai, R., 1995. A reaction-diffusion wave on the skin of the marine angelfish Pomacanthus. Nature, Volume 376, pp. 765-768.

Kondo, S. \& Miura, T., 2010. Reaction-Diffusion Model as a Framework for Understanding Biological Pattern Formation. Science, 329(5999), pp. 1616-1620.

Magnani, L., 2004. Model-Based and Manipulative Abduction in Science. Foundations of Science, 9(3), pp. 219-247.

Maini, P. K., Baker, R. E. \& Chuong, C.-M., 2006. The Turing Model Comes of Molecular Age. Science, 314(5804), pp. 1397-1398.

Maxwell, J. C., 1890. On Physical Lines of Force. In: W. D. Niven, ed. Scientific Papers of James Clerk Maxwell. Cambridge: Cambridge University Press, pp. 451-513.

Meyer, H., 1951. On the Heuristic Value of Scientific Models. Philosophy of Science, 18(2), pp. 111-123.

Morrison, M., 1999. Models as Autonomous Agents. In: M. Morgan \& M. Morrison, eds. Models as Mediators: Perspectives on Natural and Social Science. Cambridge: Cambridge University Press, pp. 38-65.

Morrison, M. \& Morgan, M., 1999. Models as Mediating Instruments. In: M. S. Morgan \& M. Morrison, eds. Models as Mediators. Cambridge: Cambridge University Press, pp. 10-37. 
Nersessian, N., 1999. Model-Based Reasoning in Conceptual Change. In: L. Magnani, N. Nersessian \& P. Thagard, eds. Model-Based Reasoning in Scientific Discovery. New York: Plenum Publishers, pp. 5-22.

Newman, S. A. \& Frisch, H. L., 1979. Dynamics of Skeletal Pattern Formation in Developing Chick Limb. Science, 205(4407), pp. 662-668.

Nickles, T., 1985. Beyond Divorce: Current Status of the Discovery Debate. Philosophy of Science, 52(2), pp. 177-206.

Nickles, T., 2006. Heuristic Appraisal: Context of Discovery or Justification?. In: J. Schickore \& F. Steinle, eds. Revisiting Discovery and Justification. Dordrecht: Springer, pp. 159-182.

Pattern Formation Group, O. U., n.d. Papers of the Pattern Formation Group. [Online] Available at: www.fbs.osaka-u.ac.jp/labs/skondo/paper_laboE.html [Accessed 3103 2017].

Pipes, L., 1953. An Operational Analysis of Traffic Dynamics. Journal of Applied Physics , 24(3), pp. 274-281.

Reutlinger, A., Hangleiter, D. \& Hartmann, S., forthcoming. Understanding (With) Toy Models. British Journal for the Philosophy of Science.

Rheinberger, H.-J., 2010. An Epistemology of the Concrete: Twentieth-Century Histories of Life. Durham, NC: Duke University Press.

Saunders, J. W., Cairns, J. M. \& Gasseling, M. T., 1957. The role of the apical ridge of ectoderm in the differentiation of the morphological structure and inductive specificity of limb parts in the chick. Journal of Morphology, 101(1), pp. 57-87.

Sheth, R. et al., 2012. Hox genes regulate digit patterning by controlling the wavelength of a Turing-type mechanism. Science, 338(6113), p. 1476-1480.

Steele, K. \& Werndl, C., forthcoming. Model-Selection Theory: The Need for a More Nuanced Picture of Use-Novelty and Double-Counting. British Journal for the Philosophy of Science.

Steinle, F., 1997. Entering New Fields: Exploratory Uses of Experimentation. Philosophy of Science, 64(Proceedings of the PSA1996, Pt. II), pp. S65-S74.

Suárez, M., 1999. The Role of Models in the Application of Scientific Theories:

Epistemological Implications. In: M. S. Morgan \& M. Morrison, eds. Models as Mediators: Perspectives on Natural and Social Science. Cambridge: Cambridge University Press, pp. 168-195.

Taylor, P., 1989. Revising Models and Generating Theory. Oikos, 54(1), pp. 121-126.

Thompson, D. W., 1917. On Growth and Form. Cambridge: Cambridge University Press. 
Turing, A., 1952. The Chemical Basis of Morphogenesis. Philosophical Transactions of the Royal Society of London. Series B, Biological Sciences, Issue 641, pp. 37-72.

Wageningen-Kessels, F. v., van Lint, H., Vuik, K. \& Hoogendoorn, S., 2015. A Genealogy of Traffic Flow Models. EURO Journal on Transportation and Logistics, 4(4), p. 445-473.

Waters, C. K., 2007. The Nature and Context of Exploratory Experimentation: An Introduction to Three Case Studies of Exploratory Research. History and Philosophy of the Life Sciences, 29(3), pp. 275-284.

Weisberg, M., 2007. Who is a Modeler?. British Journal for the Philosophy of Science, Volume 58, pp. 207-233.

Wimsatt, W. C., 2007. Re-Engineering Philosophy for Limited Beings: Piecewise Approximations to Reality. Cambridge, Mass.: Harvard University Press.

Wolpert, L., 1969. Positional Information and the Spatial Pattern of Cellular Differentiation. Journal of Theoretical Biology, 25(1), pp. 1-47.

Wolpert, L., Tickle, C. \& Martinez Arias, A., 2015. Principles of Development. 5th ed. Oxford: Oxford University Press. 